\title{
Satisfacción personal de mujeres en condiciones de vulnerabilidad de Ciudad Guzmán, Jalisco
}

\author{
Personal satisfaction of women in conditions of \\ vulnerability of Ciudad Guzman, Jalisco
}

\begin{abstract}
Claudia Saldaña ${ }^{1}$, Luis de Loera ${ }^{1}$
Forma de citar: Saldaña C, de Loera L. Satisfacción personal de mujeres en condiciones de vulnerabilidad de Ciudad Guzmán, Jalisco. Rev Univ Ind Santander Salud. 2017; 49(2); 312-318. DOI: http://dx.doi.org/10.18273/revsal.v49n2-2017004 @ (1)

\section{RESUMEN}

Introducción: Las mujeres en condiciones de vulnerabilidad de sectores específicos de la población deben lidiar con circunstancias que ponen en riesgo su autoestima y satisfacción personal con la vida que llevan. Objetivo: Evaluar los niveles de satisfacción de las mujeres en condiciones de vulnerabilidad de Ciudad Guzmán, Jalisco, a partir de la autopercepción que muestran de su situación de vida. Metodología: Estudio descriptivo correlacional llevado a cabo en junio de 2016. Participaron 122 mujeres beneficiarias de despensa, que respondieron al instrumento ISPRELEBA. Se comparó el puntaje global de insatisfacción con las condiciones de vulnerabilidad y las variantes sociodemográficas con una prueba de Chi-cuadrado de Pearson. Resultados: Los sectores vulnerables que mayores niveles de insatisfacción personal presentan son las mujeres solas o en viudez, las embarazadas y en periodo de lactancia, cuyo nivel de estudios no cubre la escuela básica. Conclusiones: Se encontraron escasas iniciativas para atender problemas emocionales de las involucradas. Se sugiere un programa de atención psicológica e integración social que estimule el progreso individual de las mujeres vulnerables.
\end{abstract}

Palabras clave: Satisfacción Personal, vulnerabilidad en salud, carencia psicosocial, autoimagen, autonomía profesional.

\begin{abstract}
Introduction: The vulnerability conditions of specific sectors of population have to deal with circumstances that jeopardize their self-concept and personal satisfaction with their lifestyle. Objective: Evaluate the satisfaction levels of women in conditions of vulnerability in Ciudad Guzman, Jalisco, according to the self-perception of their life situation. Methodology: Correlational descriptive study implemented in June 2016. 122 beneficiary women answered the ISP-RELEBA instrument. The global score of dissatisfaction was compared with the vulnerability conditions and sociodemographic variables with a Pearson's Chi-squared test. Results: The vulnerable sectors with highest personal dissatisfaction levels are lonely women and widows, followed by pregnant and breastfeeding women, whose educational levels do not cover the basic school. Conclusion: There are few initiatives to attend to the psychological problems of those involved. It is suggested a psychological assistant and social integration program which stimulate the individual progress of vulnerable women.
\end{abstract}

Keywords: Personal Satisfaction, health vulnerability, psychosocial factors, self-concept, professional autonomy.

1. Universidad de Guadalajara, Ciudad Guzmán, México.

Correspondencia: Claudia Saldaña Orozco. Dirección: López Mateos 33 (Colonia Loma Bonita, Ciudad Guzmán, México). Correo electrónico: claudias@cusur.udg.mx. Teléfono: +52 341883669 Ext. 46076. 


\section{INTRODUCCIÓN}

De acuerdo con la Organización Mundial de la Salud: "la salud de la mujer está ligada a su posición en la sociedad, se beneficia con la equidad y sufre por discriminación"1. Por su parte, Delgadillo define los factores psicosociales como:

Elementos derivados de la relación dinámica entre el individuo, el trabajo y el entorno, que afectan positiva o negativamente su estado de bienestar, acercándolo o distanciándolo de manera sostenida o intermitente al desarrollo de sus potencialidades humanas, así como a su progreso².

El funcionamiento humano y el bienestar se mantienen en un proceso de adaptación continuo, regulado por las estructuras mentales y el medio ambiente en que se desarrolla el individuo. La disponibilidad de recursos y la percepción de bienestar subjetivo son elementos que en conjunto instauran metas en la búsqueda de la satisfacción por la vida ${ }^{3}$.

A lo largo del territorio mexicano, el Sistema Nacional de Desarrollo Integral de la Familia se encarga de diseñar programas y aplicar las políticas públicas, con la intención de dar respuesta a las necesidades y demandas que en materia de asistencia social, presentan las personas y grupos sociales que más lo necesitan. Uno de los apoyos expedidos por esta organización a población en condición de pobreza, consta de una despensa alimenticia que se entrega de manera mensual, al igual que diversos programas de apoyo emocional.

El rango de vulnerabilidad de los riesgos psicosociales abarca todos los aspectos en la vida del individuo. Las amenazas de la sociedad moderna pueden relacionarse con factores como la competitividad laboral, el tráfico, el ruido, las disputas maritales, la educación de los hijos, entre otros $^{4}$. De la misma manera, entre estos factores se encuentran las dificultades económicas, la intimidad con la pareja, los acontecimientos políticos y sociales y la formación académica.

Asimismo, la integración social es un aspecto de consideración en lo que a salud se refiere. Los altos índices de salud y de bienestar están relacionados con una mayor cantidad de capital social, lo cual implica que la promoción de la salud pública debe tomar en cuenta el punto de vista de la participación en la sociedad para conseguir un impacto positivo en la calidad de vida de la población ${ }^{5}$.
En el caso de las mujeres de mediana edad, se deben tomar en cuenta otros factores sociobiológicos como el envejecimiento de los padres, la atención de los hijos, la viudez, la jubilación al igual que los cambios físicos propios de la vejez, ineludibles en dicha etapa de la vida y que contribuyen a incrementar los riesgos de vulnerabilidad y la calidad de vida ${ }^{6}$.

La administración de los recursos resulta fundamental para lidiar con la problemática de la vulnerabilidad social $^{7}$. Una limitación en los recursos económicos desemboca en una mayor vulnerabilidad, dando como resultado un estado de pobreza. Al respecto, y en relación con las oportunidades que se encuentran al alcance de las mujeres, Arriagada ha redimensionado el fenómeno de la pobreza como una derivación de acceso restrictivo a la propiedad, bajos ingresos y bajo consumo, de limitadas oportunidades sociales, políticas y laborales, así como de insuficientes logros educativos, en salud, nutrición y acceso, uso y control en materia de recursos naturales, incluyendo otras áreas del desarrollo social, lo que genera subjetividades ancladas al desamparo e impotencia, que llevan a conformar identidades centradas en la inequidad y falta de justicia, particularmente para las mujeres en estados de pobreza ${ }^{8}$.

En un estudio realizado por Arcos, et al. ${ }^{9}$ se encontró que las mujeres que trabajaban de manera informal obtenían bajos ingresos y baja cobertura de seguridad social, situación que llevaba consigo la ausencia de beneficios para el ejercicio de una maternidad segura y una infancia protegida. Por ello, la educación y capacitación de las mujeres es una vía de desarrollo que puede mejorar las condiciones de acceso al empleo y repercutir tanto en su situación individual como en el bienestar del núcleo familiar ${ }^{10}$.

Los resultados sugieren la realización operaciones efectivas de protección social, así como de trabajos interdisciplinarios a cargo de las autoridades. Con la intención de desarrollar planes de acción verdaderamente efectivos, Lamas propone intervenciones asistenciales que delimiten las áreas de acción y se concentren en alcanzar un mayor grado de justicia social y en la satisfacción de las necesidades básicas ${ }^{11}$.

El Pacto Internacional de Derechos Económicos, Sociales y Culturales, al que la mayoría de los países de América se han sumado, señala en los artículos 6 al 15 , los derechos a un trabajo digno, a la alimentación, a vestido y a una vivienda adecuada para toda persona y su familia ${ }^{12}$. En algunos países como Costa Rica, El Salvador y Panamá se ha dado estímulo a la creación 
de empresas para el fortalecimiento social y económico de las mujeres, se impulsó la creación de la unidad de equidad de género en el Ministerio de Trabajo y Seguridad Social, y se hicieron reformas en cuanto al trabajo como son los derechos en período de maternidad y una prohibición a demandar un examen de embarazo previo a contrato ${ }^{13}$.

En el caso concreto de México, en los años noventa vieron la luz múltiples iniciativas gubernamentales como el Programa de Educación, Salud y Alimentación (Progresa) y el Programa Nacional de Solidaridad (PRONASOL), que tenían como objetivos generales la erradicación de la pobreza extrema, propiciar la participación social, económica y política de las mujeres marginadas e impulsar proyectos integrales orientados a liberarlas de cargas y tiempos excesivos de trabajo.

Asimismo, el Programa de Abasto Social de Leche (PASL) es otro de los programas nacionales que opera a través de puntos de venta autorizados por Liconsa. La porción de leche autorizada es de cuatro litros a la semana por beneficiario registrado en el padrón y cada hogar tendrá derecho a adquirir un máximo de 24 litros a la semana, dependiendo del número de sus beneficiarios ${ }^{14}$.

Estas propuestas fueron generadas tomando en consideración que la mujer requiere de apoyos integrales en materia de salud y educación, así como de la gestión de sus capacidades para el desarrollo de actividades productivas que le permitan acceder a mejores niveles de bienestar con su familia ${ }^{15}$. Sin embargo, cabe hacer énfasis en las reducidas asignaciones presupuestarias para este programa, que a lo largo de seis años no recibió ni siquiera el 0,3\% de la inversión total federal en lo que a la erradicación de la pobreza se refiere.

En el municipio de Ciudad Guzmán, Jalisco, el programa de asistencia alimentaria a población vulnerable hace válido este apoyo a través del subprograma de ayuda alimentaria directa. El DIF Municipal ofrece despensas con productos básicos y charlas de orientación alimentaria que fomenten los buenos hábitos alimenticios, contribuyendo a mejorar su calidad de nutrición.

El problema radica en que se intenta superar obstáculos de bienestar colectivo desde una sola perspectiva: la económica. La calidad de vida debe ser entendida como un estado de bienestar general que se compone de factores objetivos y subjetivos, como el bienestar físico, material, social y emocional, en conjunto con el desarrollo personal y la autopercepción que el individuo tiene de sus propias condiciones ${ }^{16}$.

En el caso de las beneficiarias de la ayuda alimentaria directa en Ciudad Guzmán, es requisito fundamental que las mujeres manifiesten condiciones vulnerables para ser merecedoras del apoyo expedido. Sin embargo, dado que las beneficiarias no son participes de ningún otro programa que estimule una mejora en la calidad de vida en cuestiones emocionales, no existe el incentivo necesario para promover ese bienestar general deseado.

A partir de esta situación surge la necesidad de evaluar los niveles de satisfacción personal, entendida como:

El balance entre las expectativas (proyección de futuro) y los logros (valoración del presente) [...] en las áreas de mayor interés para el ser humano y que son el trabajo, la familia, la salud, las condiciones materiales de vida, las relaciones interpersonales, y las relaciones sexuales y afectivas con la pareja ${ }^{17}$.

De este modo, se podrá determinar la calidad del apoyo expedido, así como identificar los campos de acción que exigen atención inmediata en pro de mejorar la calidad de vida de las mujeres vulnerables de la región.

Lo anterior deja de manifiesto que, a pesar del creciente número de propuestas en materia de igualdad y pobreza, los apoyos económicos asignados por el gobierno no satisfacen los requerimientos de los mismos programas en la lucha por ver cumplidos sus objetivos. Además, es importante recalcar la escasez de programas dedicados a la atención psicológica y emocional, cuya ausencia impide el progreso equilibrado del bienestar general de los sectores vulnerables. Por lo tanto, es indispensable la recolección de evidencia que ponga de manifiesto la situación crítica de vulnerabilidad a la que están sometidas miles de mujeres, y que no reciben la atención necesaria para superar las condiciones tan precarias en las que están impuestas.

\section{METODOLOGÍA}

Se llevó a cabo un estudio cuantitativo de carácter descriptivo correlacional, que pretende encontrar la asociación entre mujeres en condición de vulnerabilidad, representadas en este caso por beneficiarias de despensas del DIF, y sus niveles de satisfacción personal. Por lo tanto, cabe preguntarse cuál es la autopercepción que las mujeres vulnerables 
tienen de sus condiciones de vida y cómo afecta en sus niveles de satisfacción. La muestra del estudio se integró por 112 mujeres, seleccionadas de la base de datos proporcionada por el DIF Municipal mediante muestreo simple aleatorio. Se expidió el apoyo a un total de 785 beneficiarios, de los cuales 741 fueron mujeres, que componen el universo de la investigación.

Se empleó el instrumento ISP-RELEBA, de Díaz Llanes, Alonso González y Bayarre Vea, diseñado por la Facultad de Salud Pública y validado en la población de mujeres de edad mediana del policlínico Cristóbal Labra de La Habana, $\mathrm{Cuba}^{18}$. Éste evalúa la satisfacción personal en la mujer de edad mediana, conforme a cinco predictores de bienestar. Las respuestas obtenidas, consistentes en valores numéricos, son sumadas para obtener un puntaje que arroja uno de los siguientes resultados: Satisfecha (de 90 a 69 puntos); Medianamente satisfecha (de 68 a 46 puntos); Poco satisfecha (de 45 a 23 puntos); Insatisfecha (de 22 a 0 puntos).

La aplicación del instrumento se realizó de forma voluntaria y se informó a las participantes que el uso de su información personal sería completamente confidencial. Se midieron variables sociodemográficas como la edad y la escolaridad, así como el estado de vulnerabilidad determinado por el propio DIF.

Se llevó a cabo un análisis descriptivo de las variables obtenidas, cuyos datos fueron expresados en tablas de frecuencias, de acuerdo a su relevancia. Se establecieron relaciones entre las variables y el puntaje final de satisfacción proporcionado por el instrumento a través de una prueba de Chi-cuadrado de Pearson. El valor significativo se estableció en $p<0,05$. El cálculo estadístico fue realizado con el programa SPSS versión 15.0.

\section{RESULTADOS}

Las variables sociodemográficas de las participantes, tomadas en cuenta para la aplicación del instrumento, se muestran en la Tabla 1.

Como puede observarse, la mayoría de las encuestadas presenta una condición vulnerable de soledad o viudez con el 36,6\%; le siguen las mujeres embarazadas con el $24 \%$ y aquellas en periodo de lactancia con el $17 \%$. En lo que refiere a escolaridad, $34,8 \%$ tuvo la primaria como máximo nivel de estudios; a continuación, se encuentra el nivel secundaria con $28,6 \%$, mientras que $19,6 \%$ no tuvo ninguna escolaridad.
Tabla 1. Variables de las participantes.

\begin{tabular}{ccc}
\hline Variable & Frecuencia & Porcentaje \\
\hline Participantes & 129 & $100 \%$ \\
\hline Vulnerabilidad \\
Eola o en viudez & 41 & $36,6 \%$ \\
En periodo de lactancia & 27 & $24,1 \%$ \\
Discapacidad & 19 & $17 \%$ \\
Anciana & 18 & $6,3 \%$ \\
& Total & $16,1 \%$ \\
& $\mathbf{1 0 0} \%$ \\
\hline Ninguna & 22 & \\
Primaria & 39 & $19,6 \%$ \\
Secundaria & 32 & $34,8 \%$ \\
Preparatoria & 14 & $28,6 \%$ \\
Técnica & 5 & $12,5 \%$ \\
& Total & $4,5 \%$ \\
\hline
\end{tabular}

Tomando como referencia algunos reactivos de la Tabla 2 , se puede apreciar que más del $50 \%$ de las encuestadas se ven afectadas por los síntomas de la menopausia. A su vez, el $52.3 \%$ considera que sería feliz al compartir la vida con una pareja. Paralelamente, el $69.2 \%$ asegura que casi siempre se sienten satisfechas con la familia que han formado; por otro lado, más del $70 \%$ afirma que, en ocasiones, se sienten irritables pero no logran explicar la razón. Se encontró también que más del 70\% de las participantes aseguran fatigarse constantemente por los quehaceres del hogar, a pesar de que el mismo porcentaje afirma que dichos quehaceres son repartidos entre los miembros de la familia.

Tabla 2. Reactivos del instrumento.

\begin{tabular}{ccccc}
\hline Reactivos y factores & $\begin{array}{c}\text { Casi } \\
\text { siempre } \\
(\%)\end{array}$ & $\begin{array}{c}\text { A veces } \\
\mathbf{( \% )}\end{array}$ & $\begin{array}{c}\text { Casi nunca } \\
(\%)\end{array}$ & $\begin{array}{c}\text { Nunca } \\
(\%)\end{array}$ \\
\hline $\begin{array}{c}\text { Los síntomas de las } \\
\text { menopausia afectan mi } \\
\text { vida }\end{array}$ & $(27.7 \%)$ & $(25.4 \%)$ & $(11.5 \%)$ & $(35.4 \%)$ \\
$\begin{array}{c}\text { Me haría feliz compartir } \\
\text { la vida con una pareja }\end{array}$ & $(52.3 \%)$ & $(26.9 \%)$ & $(6.9 \%)$ & $(13.8 \%)$ \\
$\begin{array}{c}\text { Me siento satisfecha } \\
\text { con la familia que he } \\
\text { logrado formar }\end{array}$ & $(69.2 \%)$ & $(22.3 \%)$ & $(2.3 \%)$ & $(6.2 \%)$ \\
$\begin{array}{c}\text { Me siento irritable e } \\
\text { inquietable aunque no sé } \\
\text { explicar lo que me pasa }\end{array}$ & $(23.8 \%)$ & $(58.5 \%)$ & $(9.2 \%)$ & $(8.5 \%)$ \\
$\begin{array}{c}\text { Las responsabilidades } \\
\text { domésticas y familiares } \\
\text { logran agotarme }\end{array}$ & $(36.2 \%)$ & $(43.8 \%)$ & $(10.8 \%)$ & $(9.2 \%)$ \\
$\begin{array}{c}\text { En mi casa el trabajo } \\
\text { doméstico se comparte } \\
\text { entre todos }\end{array}$ & $52(40 \%) 52(40 \%)$ & $17(13.1 \%) 9(6.9 \%)$ \\
\hline
\end{tabular}


Respecto al nivel general de satisfacción personal, el $56,3 \%$ de las participantes se cataloga como medianamente satisfecha, mientras que el 31,3\% dice estar poco satisfecha. No se encontró un porcentaje de mujeres insatisfechas. En la Tabla 3, sobre aquellas poco satisfechas, se puede apreciar que las mujeres solas o en viudez representan $31,4 \%$, mientras que aquellas que se encuentran embarazadas o en periodo de lactancia se mantienen con $28,6 \%$. Por su parte, el nivel de educación secundaria conserva el mayor porcentaje de poca satisfacción con $37,1 \%$, seguidos por el nivel primaria con $34,3 \%$.

Tabla 3. Cifras de mujeres poco satisfechas.

\begin{tabular}{ccc}
\hline & Vulnerabilidad & \\
\hline & Poco satisfechas & Frecuencia \\
\hline Sola o en viudez & $31,4 \%$ & 11 \\
Embarazada & $28,6 \%$ & 10 \\
Discapacidad & $2,9 \%$ & 1 \\
Lactancia & $28,6 \%$ & 10 \\
Anciana & $8,6 \%$ & 3 \\
Total & $\mathbf{1 0 0 \%}$ & $\mathbf{3 5}$ \\
\hline & Escolaridad & \\
\hline Ninguna & $14,3 \%$ & 5 \\
Primaria & $34,3 \%$ & 12 \\
Secundaria & $37,1 \%$ & 13 \\
Preparatoria & $10,4 \%$ & 4 \\
Técnica & $2,9 \%$ & 1 \\
Total & $\mathbf{1 0 0} \%$ & $\mathbf{3 5}$ \\
\hline
\end{tabular}

Tabla 4. Prueba de chi-cuadrado de Person entre satisfacción y vulnerabilidad.

\begin{tabular}{lccc}
\hline Chi-cuadrado de Pearson & Valor & gl & $\begin{array}{c}\text { Sig. asintótica } \\
\text { (bilateral) }\end{array}$ \\
\cline { 2 - 4 } & $16,163^{\mathrm{a}}$ & 8 & 0,040 \\
\cline { 1 - 3 } Razón de verosimilitudes & 17,018 & 8 & 0,030 \\
Lineal & 0,184 & 1 & 0,668 \\
N de casos válidos & \multicolumn{3}{c}{129} \\
\hline
\end{tabular}

a 5 casillas $(33,3 \%)$ tienen una frecuencia esperada inferior a 5 .

La frecuencia mínima esperada es 1,12 .

En la Tabla 4, La prueba de chi-cuadrado de Pearson demuestra una significación asintótica bilateral de 0,04 entre los niveles de satisfacción y los estados de vulnerabilidad de las encuestadas, mientras que entre el grado de escolaridad, mostrado en la Tabla 5, y la satisfacción no arrojó resultados significativos. Igualmente, la prueba de regresión lineal de la Figura 1 no demostró una relación significativa entre la satisfacción personal y las edades de las participantes.

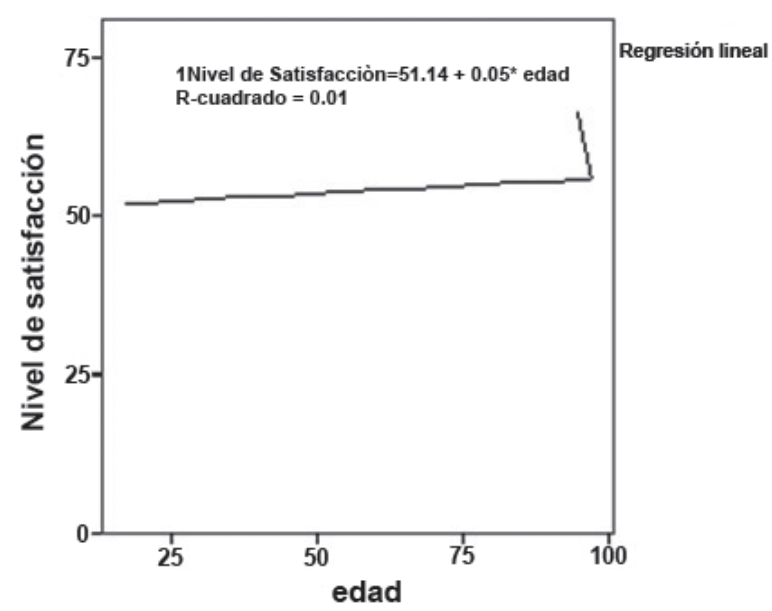

Figura 1. Regresión lineal entre el nivel de satisfacción y la edad.

Tabla 5. Prueba de chi-cuadrado de Person entre satisfacción y escolaridad.

\begin{tabular}{cccc}
\hline \multirow{2}{*}{ Chi-cuadrado de Pearson } & Valor & gl & $\begin{array}{c}\text { Sig. asintótica } \\
\text { (bilateral) }\end{array}$ \\
\cline { 2 - 4 } & $5,459^{\mathrm{a}}$ & 8 & 0,708 \\
\hline Razón de verosimilitudes & 5,482 & 8 & 0,705 \\
Lineal & 1,582 & 1 & 0,208 \\
N de casos válidos & & \multicolumn{2}{c}{129} \\
\hline
\end{tabular}

a 7 casillas $(46,7 \%)$ tienen una frecuencia esperada inferior a 5. La frecuencia mínima esperada es 0,62.

\section{DISCUSIÓN}

De acuerdo a los resultados obtenidos, el sector vulnerable más amplio de la muestra lo constituyen las mujeres solas o en estado de viudez, seguidas de cerca por las mujeres embarazadas y en periodo de lactancia. En lo que corresponde al nivel de estudios, el $85 \%$ apenas alcanzó la escuela básica, y algunas de ellas no tuvieron ningún grado de estudios en absoluto. Esto indica la premura de atender las carencias educativas con la intención de impulsar a este sector vulnerable en particular, a instancias superiores en su nivel educativo.

Respecto del nivel de satisfacción, las mujeres solas o en viudez, junto con las embarazadas y en periodo de lactancia, constituyen el sector menos satisfecho con sus condiciones de vida, siendo que el $30 \%$ en cada caso mostraron un nivel de poca satisfacción. Si bien una gran parte se proclama satisfecha con la familia que ha logrado formar, los niveles de insatisfacción se concentran en los sectores correspondientes a la vida en pareja. De lo anterior se deduce que mantener una buena relación conyugal contribuye a una percepción positiva del bienestar propio. 
A propósito, el aspecto emocional resulta crucial para propiciar altos índices de satisfacción personal. Uno de los reactivos arrojó como resultado que más del setenta por ciento de las encuestadas se sienten irritables sin lograr explicar la razón.

La mayoría de los programas gubernamentales (como el PRONASOL o el Programa de Desarrollo Productivo de la Mujer, mencionados anteriormente) que apoyan a los sectores vulnerables, se concentran en satisfacer necesidades económicas, dejando de lado aspectos psicológicos. Por esta razón, las propuestas de intervención que se originen a partir de estos resultados deben enfocarse en atender la salud mental de las mujeres, y preocuparse por aminorar los riesgos psicosociales que amenazan su bienestar integral.

Un aspecto que llama la atención es el agotamiento que provocan las labores domésticas. Esto indica que, a pesar de que las labores no están por completo a su cargo, la responsabilidad (reforzada por la idea de que la mujer debe hacerse cargo del hogar) recae sobre ellas, lo cual representa otro campo de acción que debe ser atendido por la atención psicológica, tentativamente por terapia familiar.

A partir de los resultados obtenidos en la investigación, se concluyó que el estado de soledad, por sí solo, puede considerarse como un factor de riesgo que a la larga producirá malestares psicológicos y consecuentemente producir trastornos emocionales. Por ello, se exhorta a las autoridades pertinentes a realizar acciones en pro de la salud mental a nivel individual, familiar y colectivo. La atención psicológica y los programas de integración social son oportunidades viables para hacer que las mujeres reconsideren sus condiciones de vida y se encaminen a elevar su autoestima.

De la misma manera, otro de los sectores que presentó mayor vulnerabilidad es el de las mujeres embarazadas o en periodo de lactancia. Un apoyo asistencial en esta etapa tan susceptible a riesgos emocionales es fundamental para incrementar el nivel de satisfacción personal, al igual que su salud psicológica y sus relaciones interpersonales.

Por otro lado, el bajo nivel de escolaridad coloca a las participantes en una posición aún más vulnerable, siendo que esta situación limita la posibilidad de obtener estabilidad laboral y condiciona su disposición al emprendimiento, iniciativa de superación personal y crecimiento tanto intelectual como social.
En conclusión, los sectores vulnerables que mayores niveles de insatisfacción personal presentan son las mujeres solas o en viudez, las embarazadas y en periodo de lactancia, cuyo nivel de estudios no cubre la escuela básica. Los aspectos relacionados con la vida en pareja causan mayor impacto negativo en su autoestima, por lo que un programa de atención psicológica e integración social, que dedique la atención necesaria para atender a mujeres solas o embarazadas, es crucial para estimular el progreso individual de las mujeres vulnerables.

\section{AGRADECIMIENTOS}

Los autores agradecen a la institución del DIF Municipal por su disposición y a los alumnos de la Licenciatura en Negocios Internacionales por su colaboración.

\section{CONFLICTO DE INTERESES}

Los autores no encontraron ningún conflicto de interés.

\section{REFERENCIAS}

1. Lazarevich I, Mora-Carrasco F. Depresión y género: Factores psicosociales de riesgo. Sea ÉPoca. 2008; 114): 7-16.

2. Uribe Prado JF. Justicia social, trabajo y factores psicosociales. En: Uribe Prado JF, Clima y ambiente organizacional. México: El Manual Moderno; 2014: p. 8-34.

3. Arita Watanabe BY. Satisfacción por la vida y teoría homeostática del bienestar. Psicol Salud. 2005; 15(5): 121-126.

4. Sandín B. El estrés: un análisis basado en el papel de los factores sociales. Inl F Clin Health Psychol. 2003; 331): 141-157.

5. Herrera PonceMS, Elgueta Rosas RP,FernándezLorca MB. Capital social, participación en asociaciones y satisfacción personal de las personas mayores en Chile. Rev Sáude Pública. 2014; 48(8): 739-749. DOI:10.1590/S0034-8910.2014048004759.

6. Yanes Calderón M, Chio Naranjo I, Pérez Felpeto R. Satisfascción personal de la mujer de edad mediana y su relación con otros factores de la vida cotidiana. Rev Cubana Obstet Ginecol. 2012; 38(8): 199-213.

7. Pizarro R. La vulnerabilidad social y sus desafíos: una mirada desde América Latina. Santiago de Chile: Publicación de las Naciones Unidas; 2001.

8. Flores Palacios F. Vulnerabilidad y representación social de género en mujeres de una comundidad migrante. Península. 2014; 992): 41-58.

9. Arcos E, Muñoz LA, Sánchez X, Vollrath A, Latorre C, Bonatti C J. Vulnerabilidad social 
en mujeres embarazadas de una comuna de la región metropolitana. Rev Méd Chile. 2011; 13): 739-747. DOI: http://dx.doi.org/10.4067/S003498872011000600007.

10. Horbath JE, Gracia A. Discriminación laboral y vulnerabilidad de las mujeres frente a la crisis mundial en México. Econ Soc Territ. 2014; 14145): 465-495.

11. Gómez E, Muñoz MM, Haz AM. Familias multiproblemáticas $\mathrm{y}$ en riesgo social: características e intervención. Psykhe. 2007; 16(2): 43-54. DOI: http://dx.doi.org/10.4067/S071822282007000200004.

12. Palacio Lavín AR. El Pacto Internacional de Derechos Económicos Sociales y Culturales (Fascículo 4). México: Comisión Nacional de los Derechos; 2012.

13. García M. Estudio sobre la autonomía económica de las mujeres en Costa Rica, el Salvador y Panamá. México: CEPAL, 2015.

14. Secretaria de Desarrollo Social. Diagnóstico integral de los programas alimentarios de la SEDESOL. México: SEDESOL; 2012.

15. Núñez García S. Género y programas de combate a la pobreza en México: ¿reconocimiento del capital social? En: Atria R, Siles M, Arriagada I, Robinson LJ, Whiteford S, Capital social y reducción de la pobreza en América Latina y el Caribe: en busca de un nuevo paradigma. Santiago de Chile: Publicación de las Naciones Unidas, 2003: p. 379-394.

16. Urzúa A, Caqueo-Urízar A. Calidad de vida: Una revisión teórica del concepto. Ter Psicol. 2012; 30(1): 61-71. DOI: http://dx.doi.org/10.4067/ S0718-48082012000100006.

17. Victoria García-Viniegras CR, González Benítez I. La categoría bienestar psicológico. Su relación con otras categorías sociales. Rev Cubana Med Gen Integr. 2000; 16(6): 586-592.

18. Díaz Llanes G, Alonso González R, Bayarre Vea H. Prevalencia de Insatisfacción Personal en Mujeres de Edad Mediana del Policlínico "Cristóbal Labra". Rev Cubana Salud Pública. 2000; 26(2): 101-109. 